Check for updates

Cite this: Phys. Chem. Chem. Phys. 2019, 21, 2196

DOI: $10.1039 / c 9 c p 90011 j$

rsc.li/pccp

\section{Correction: 3T1R model and tuning of thermoluminescence intensity by optimization of dopant concentration in monoclinic $\mathrm{Gd}_{2} \mathrm{O}_{3}: \mathrm{Er}^{3+} ; \mathrm{Yb}^{3+} \mathrm{co}$-doped phosphor}

\author{
Raunak Kumar Tamrakar, ${ }^{a}$ Kanchan Upadhyay ${ }^{\star b}$ and D. P. Bisen ${ }^{c}$
}

Correction for '3T1R model and tuning of thermoluminescence intensity by optimization of dopant concentration in monoclinic $\mathrm{Gd}_{2} \mathrm{O}_{3}: \mathrm{Er}^{3+} ; \mathrm{Yb}^{3+}$ co-doped phosphor' by Raunak Kumar Tamrakar et al., Phys. Chem. Chem. Phys., 2017, 19, 14680-14694.

In this PCCP paper, the authors used a model previously published by R. Chen et al. in Radiation Measurements. The missing reference is listed herein as ref. 1 , and the authors apologise that it was not clear in the paper that the model was originally proposed by Chen et al. or cited in the PCCP paper. The model proposed in ref. 1 is a general model to explain the concentration quenching. behaviour of thermoluminescence. In the $P C C P$ paper, the authors used this model to explain the concentration quenching behaviour of the $\mathrm{Gd}_{2} \mathrm{O}_{3}: \mathrm{Er}^{3+} ; \mathrm{Yb}^{3+}$ co-doped phosphor. Equations 1-21 in the PCCP paper have been reproduced from ref. 1.

The following corrections are implemented including citations to ref. 1 in the Introduction, Results and discussion and Conclusion sections:

On page 14681, the sentence beginning "Furthermore, we used Chen's peak shape method..." should be changed to "Furthermore, we used Chen's peak shape method and glow curve deconvolution (GCD) functions ${ }^{1}$ to analyse the TL curve and hence calculated the trapping parameters for $\mathrm{Er}^{3+}$ and $\mathrm{Yb}^{3+}$-co-doped $\mathrm{Gd}_{2} \mathrm{O}_{3}$."

On page 14688, the sentence beginning "As the present study..." should be changed to "As the present study deals with the tuning of the thermoluminescence behaviour of the $\mathrm{Gd}_{2} \mathrm{O}_{3}: \mathrm{Er}^{3+} ; \mathrm{Yb}^{3+}$ phosphor with dopant concentration, we used Chen's model ${ }^{1}$ to explain the effect of concentration quenching."

On page 14693, the last two sentences of the conclusions should be changed to "Moreover, three trap and one recombination centre model ${ }^{1}$ was used to explain the concentration quenching effect on the thermoluminescence behaviour of the prepared sample. The experimental results show that the peak at lower temperature reaches the maxima before the second peak, resembling Chen's theoretical model used herein for the sample."

The authors also apologise for portions of unattributed text overlap in the Results and discussion and Conclusion sections with the authors' previous work, including ref. 11, 13, 16, 19 and 21 from the PCCP article, and an uncited article listed herein as ref. 2. In spite of the text overlap, new data has been reported in this $P C C P$ article, which was not reported in the references listed above.

The Royal Society of Chemistry apologises for these errors and any consequent inconvenience to authors and readers.

\title{
References
}

1 R. Chen, J. L. Lawless and V. Pagonis, Radiat. Meas., 2011, 46, 1380-1384.

2 R. K. Tamrakar and K. Upadhyay, J. Mater. Sci.: Mater. Electron., 2017, 28, 4267-4278.

\footnotetext{
${ }^{a}$ Department of Applied Physics, Bhilai Institute of Technology (Seth Blakrishan Memorial), Durg (C.G.), 491001, India

${ }^{b}$ Department of Chemistry, Shri Shankaracharya Vidyalaya, Hudco, Bhilai (C.G.), India. E-mail: kanchan.chemistry@gmail.com

${ }^{c}$ School of Studies in Physics and Astrophysics, Pt. Ravishankar Shukla University, Raipur (C.G.), Pin-492010, India
} 